\title{
Inclusion of photoprotective parameters in photosynthesis-measuring systems to improve the interpretation of photosynthesis and productivity
}

\author{
C.C. OGBAGA*,+ and H.U.R. ATHAR ${ }^{* *}$ \\ Department of Biological Sciences, Nile University of Nigeria, Airport Road, Abuja, Nigeria* \\ Institute of Pure and Applied Biology, Bahauddin Zakariya University, Multan, Pakistan **
}

While reading the paper by Vredenberg (2018) published in Photosynthetica (10 January, 2018), I was inspired to write this letter.

Plant stress is any condition that can negatively affect the morphology, physiology, and biochemistry of plants (Ogbaga et al. 2014). Such stress can be biotic or abiotic, induced by pests and non-living factors, such as salinity, drought, etc. Plants adopt various mechanisms to respond and adapt to such stresses (Ogbaga et al. 2014). Physiologically, stress can be assessed with the use of photosynthesis-measuring systems. Monitoring the degree of stress in crops at early growth stage and timely remedy is essential, otherwise a substantial loss in crop productivity may occur (Ogbaga et al. 2018). Conventional methods for crop monitoring are generally time-consuming or involve invasive methods. Similarly, various physiological indicators for selecting stress-tolerant species in breeding programs are also based on invasive techniques.

In the past 40 years, various researchers suggested the use of different chlorophyll (Chl) fluorescence parameters for identifying stress-tolerant cultivars. However, some of them disagree on parameter suitability (a long list of references is given in Desktop Plant Stress Guide Edition 5.0 https://optisci.com/assets/basic plant_stress guide v5.pdf, accessed 25 October, 2018). Little information is available for general users to decide which Chl fluorescence protocol and parameter is the most suitable for screening and selection of stress tolerance. Since 1996, the role of nonphotochemical quenching (NPQ) and cyclic electron transport chain have been significantly studied in stress tolerance (Demmig-Adams and Adams 1996, Niyogi 1999, D'Ambrosio et al. 2008, Guadagno et al. 2010, Einali et al. 2012, Kromdijk et al. 2016, Lima Neto et al. 2017, Murchie 2017).

Murchie and Niyogi (2011) suggested the manipulation of photoprotective components of NPQ to improve photosynthetic efficiency and crop productivity. Recently,
Kromdijk et al. (2016) showed an improved crop productivity by manipulating photoprotective mechanism in tobacco. In addition, Goss and Lepetit (2015) demonstrated wide biodiversity in NPQ components in different plant species which suggest that stress-tolerant cultivars can be identified using photoprotective components. In parallel to this development, various researchers have proposed the correct way of calculating NPQ components (Maxwell and Johnson 2000, Guadagno et al. 2010, Roháček 2010, Kasajima et al. 2015, Tietz et al. 2017) that can be used for identifying stress-tolerant cultivars or in assessing efficiency of photoprotective components of NPQ in stress tolerance (Ruban 2016, Giovagnetti et al. 2018).

It is well established that NPQ has two main components: 1) fast relaxation $\left(\mathrm{NPQ}_{\mathrm{f}}\right)$, i.e., $\mathrm{q}_{\mathrm{E}}$ and 2) slow relaxation $\left(\mathrm{NPQ}_{\mathrm{s}}\right)$, i.e., $\mathrm{q}_{\mathrm{I}}$. Available published reports suggest that the separation of these components requires detailed understanding of mechanism, technique, and algorithms.

Photosynthesis-measuring systems have been evolved over the years and are currently set up by default to measure Chl $a$ fluorescence and gas exchange. General photosynthesis-measuring instruments have default protocols with specific calculated parameters. Often, in order to calculate the parameter of interest, users have to do this by themselves and it requires detailed understanding of protocols and algorithms. Some scientists may help non-specialists calculate it (e-mail/ personal communications with Bernardo Duarte, Marine and Environmental Sciences Centre, Faculty of Sciences, University of Lisbon, Portugal). Some researchers provide services on commercial basis (www.fluoromatics.com) or develop supporting software such as Biolyzer v. 5 compatible with most of fluorometers. However, this company and software was closed in 2015. In view of this information, it is important to include various potential parameters for identification of stress tolerance, such as photoprotective components of NPQ, into photosynthesis-

\footnotetext{
Received 28 September 2018, accepted 25 October 2018.

${ }^{+}$Corresponding author; phone: +2349030333632, e-mail: chukwumaogbaga@gmail.com

The authors declare that they have no conflict of interest.

Abbreviations: $\mathrm{F}_{\mathrm{m}}$ - maximal fluorescence yield of the dark-adapted state; $\mathrm{F}_{\mathrm{m}}^{\circ}$ - maximum fluorescence at the dark-adapted state; $\mathrm{F}_{\mathrm{m}}{ }^{-}-$ maximum fluorescence in the light; $\mathrm{F}_{\mathrm{m}}^{\mathrm{r}}-\mathrm{F}_{\mathrm{m}}$ in the presence of slowly relaxing quenching; $\mathrm{NPQ}$ - nonphotochemical quenching; $N P Q_{\mathrm{f}}$ - fast nonphotochemical quenching; $\mathrm{NPQ}_{\mathrm{s}}$ - slow nonphotochemical quenching; $\mathrm{q}_{\mathrm{E}}$ - fast component of NPQ; $\mathrm{q}_{\mathrm{I}}-$ slow component of $\mathrm{NPQ} ; \mathrm{q}_{\mathrm{T}}$ - state transitions; $\mathrm{q}_{\mathrm{z}}$ - zeaxanthin dependent quenching.
} 
measuring systems. Inclusion of such parameters would help plant breeders or non-specialists in phenotyping using Chl fluorescence (Kromdijk et al. 2016, Ruban 2016).

Popular photosynthesis-measuring systems are Licor 6400/6800 (Licor, Nebraska, USA), CIRAS-1, 2, 3 (PP Systems, Massachusetts, USA), Walz PAM systems (Heinz Walz, Effeltrich, Germany), FluorPen FP 100/110 (Photon System Instruments, Drásov, Czech Republic), and recently MultispeQ v. 2.0 from David Kramer's laboratory (Michigan, USA). None of these machines calculates $\mathrm{q}_{\mathrm{E}}$ and $\mathrm{q}_{\mathrm{I}}$ on-the-spot. Users are advised to do the calculation at their own convenience. Walz PAM systems provide $\mathrm{Y}_{\text {(NO) }}$ quantum yield of non-regulated heat dissipation, while MultispeQ v. 2.0 calculates $\mathrm{NPQ}_{\mathrm{t}}$ as photoprotective NPQ. The only photosynthesis-measuring system, which measures the parameters, is the fluorescence meter OSP5 (Opti-Sciences, New Hampshire, USA).

Licor, for instance, currently uses a pulse amplitude modulator (PAM) fluorometer for measuring $F_{0}, F_{m}, F, F_{m}$, and $\mathrm{F}_{0}{ }^{\prime}$, whilst parameters, such as $\mathrm{F}_{\mathrm{v}}, \mathrm{F}_{\mathrm{v}} / \mathrm{F}_{\mathrm{m}}, \mathrm{F}_{\mathrm{v}}{ }^{\prime} / \mathrm{F}_{\mathrm{m}}{ }^{\prime}, \Phi_{\mathrm{PSII}}, \mathrm{q}_{\mathrm{P}}$, $\mathrm{q}_{\mathrm{N}}, \mathrm{NPQ}$, and ETR are calculated (https://www.licor.com/ documents/ifuhfcjga0wvh94lkysz, accessed September 9,2018). These measurements can be performed on both dark- and light-adapted leaves. Since NPQ is calculated in the current version, it should be possible to add the calculation of fast and slow relaxation kinetic parameters using the formulae given by Maxwell and Johnson (2002): $N P Q_{\mathrm{s}}=\left(\mathrm{F}_{\mathrm{m}}^{\mathrm{o}}-\mathrm{F}_{\mathrm{m}}^{\mathrm{r}}\right) / \mathrm{F}_{\mathrm{m}}^{\mathrm{r}}$,

$N P Q_{F}=\left(F_{m}^{o} / F_{m}^{\prime}\right)-\left(F_{m}^{o} / F_{m}^{r}\right)$,

where $\mathrm{F}_{\mathrm{m}}^{\mathrm{o}}$ is the maximum fluorescence at the dark-adapted state, $\mathrm{F}_{\mathrm{m}}^{\prime}$ is the maximum fluorescence in the light, and $\mathrm{F}_{\mathrm{m}}^{\mathrm{r}}$ is $F_{m}$ in the presence of slowly relaxing quenching. This makes measurements more convenient for users interested in distinguishing fast relaxation quenching $\left(\mathrm{q}_{\mathrm{E}}\right)$ from slow quenching $\left(\mathrm{q}_{\mathrm{I}}\right)$ which is indicative of photoinhibition. It would be also useful particularly in situations where users must collect massive data in the field.

Currently, NPQ estimates and its associated components $\left(\mathrm{q}_{\mathrm{E}}, \mathrm{q}_{\mathrm{I}}, \mathrm{q}_{\mathrm{Z}}\right.$, and $\left.\mathrm{q}_{\mathrm{T}}\right)$ are derived from PAM measurements of Chl fluorescence yield; in order to obtain the maximal yield of fluorescence, leaves have to be fully dark-adapted $\left(F_{\mathrm{m}}\right)$ (Tietz et al. 2017). This approach implies a prolonged dark acclimation and prevents highthroughput use particularly under field conditions (Tietz et al. 2017). It also introduces artefacts when $\mathrm{F}_{\mathrm{m}}$ is measured in the presence of photodamaged centres (Tietz et al. 2017). Hence, separation of the contributions of NPQ and inclusion in photosynthesis-measuring systems is crucial for plant phenotyping. Thus, the development of new parameters and protocols should be brought into focus in a subsequent new generation of fluorometers.

\section{References}

D'Ambrosio N., Guadagno C.R., Virzo De Santo A.: Is qE always the major component of non-photochemical quenching? - In: Allen J., Gantt E., Golbeck J.H., Osmond, B. (ed.): Photosynthesis. Energy from the Sun. Pp. 1001-1004.
Springer, Netherlands 2008.

Demmig-Adams B., Adams W.W.: The role of xanthophyll cycle carotenoids in the protection of photosynthesis. - Trends Plant Sci. 1: 21-26, 1996.

Einali A., Shariati M., Sato F., Endo T.: Cyclic electron transport around photosystem I and its relationship to nonphotochemical quenching in the unicellular green alga Dunaliella salina under nitrogen deficiency. - J. Plant Res. 126: 179-186, 2012.

Giovagnetti V., Han G., Ware M.A. et al.: A siphonous morphology affects light-harvesting modulation in the intertidal green macroalga Bryopsis corticulans (Ulvophyceae). - Planta 247: 1293-1306, 2018.

Goss R., Lepetit B.: Biodiversity of NPQ. - J. Plant Physiol. 172: 13-32, 2015.

Guadagno C.R., Virzo De Santo A., D'Ambrosio N.: A revised energy partitioning approach to assess the yields of nonphotochemical quenching components. - BBA-Bioenergetics 1797: 525-530, 2010.

Kasajima I., Suetsugu N., Wada M., Takahara, K.: Collective calculation of actual values of non-photochemical quenching from their apparent values after chloroplast movement and photoinhibition. - Am. J. Plant Sci. 6: 1792-1805, 2015.

Kromdijk J., Głowacka K., Leonelli L. et al.: Improving photosynthesis and crop productivity by accelerating recovery from photoprotection. - Science 354: 857-861, 2016.

Lima Neto M.C., Cerqueira J.V.A., da Cunha J.R. et al.: Cyclic electron flow, NPQ and photorespiration are crucial for the establishment of young plants of Ricinus communis and Jatropha curcas exposed to drought. - Plant Biol. 19: 650659, 2017.

Maxwell K., Johnson G.N.: Chlorophyll fluorescence - a practical guide. - J. Exp. Bot. 51: 659-668, 2000.

Murchie E.H.: Safety conscious or living dangerously: what is the 'right' level of plant photoprotection for fitness and productivity? - Plant Cell Environ. 40: 1239-1242, 2017.

Murchie E.H., Niyogi K.K.: Manipulation of photoprotection to improve plant photosynthesis. - Plant Physiol. 155: 86-92, 2011.

Niyogi K.K.: Photoprotection revisited: genetic and molecular approaches. - Annu. Rev. Plant Biol. 50: 333-359, 1999.

Ogbaga C.C., Stepien P., Athar H.U.R., Ashraf, M.: Engineering Rubisco activase from thermophilic cyanobacteria into hightemperature sensitive plants. - Crit. Rev. Biotechnol. 38: 559$572,2018$.

Ogbaga C.C., Stepien P., Johnson G.N.: Sorghum (Sorghum bicolor) varieties adopt strongly contrasting strategies in response to drought. - Physiol. Plantarum 152: 389-401, 2014.

Roháček K.: Method for resolution and quantification of components of the non-photochemical quenching $\left(\mathrm{q}_{\mathrm{N}}\right)$. Photosynth. Res. 105: 101-113, 2010.

Ruban A.V.: Nonphotochemical chlorophyll fluorescence quenching: Mechanism and effectiveness in protecting plants from photodamage. - Plant Physiol. 170: 1903-1916, 2016.

Tietz S., Hall C.C, Cruz J.A., Kramer, D.M.: NPQ (T): a chlorophyll fluorescence parameter for rapid estimation and imaging of non-photochemical quenching of excitons in photosystemII-associated antenna complexes. - Plant Cell Environ. 40: 1243-1255, 2017.

Vredenberg W.J.: On the quantitative relation between dark kinetics of NPQ-induced changes in variable fluorescence and the activation state of the $\mathrm{CF}_{0} \cdot \mathrm{CF}_{1} \cdot$ ATPase in leaves. Photosynthetica 56: 139-149, 2018.

(C) The authors. This is an open access article distributed under the terms of the Creative Commons BY-NC-ND Licence. 